\title{
Efeito da atividade esportiva sistematizada sobre o desenvolvimento motor de crianças de sete a 10 anos
}

CDD. 20.ed. 152.3

613.7

http://dx.doi.org/10.1590/1807-55092015000300497

\author{
Camila Ramos dos SANTOS* \\ Carla Cristiane da SILVA** \\ Mara Laiz DAMASCENO*** \\ J osiane MEDINA-PAPST ${ }^{*}$ \\ Inara MARQUES*
}

*Centro de Educação Física e Esporte, Universidade Estadual de Londrina.

${ }^{*}$ Centro de Ciências da Saúde, Universidade Estadual do Norte do Paraná.

***Faculdade Estácio de Sá.

\section{Resumo}

Atividades físicas programadas na infância são reconhecidas por ocasionar mudanças nos diversos aspectos do desenvolvimento das crianças. Assim, o presente estudo objetivou verificar o impacto da atividade esportiva programada de ballet clássico e de futsal sobre indicadores de motricidade global e de equilíbrio em crianças. A amostra foi composta por 160 crianças entre sete e 10 anos de idade. Oitenta crianças de ambos os sexos foram selecionadas no ambiente escolar e compuseram os grupos de escolares, caracterizado pela prática exclusiva de Educação Física escolar. Os grupos vinculados à prática esportiva foram compostos por 40 crianças do sexo feminino, praticantes de "ballet" clássico e 40 do sexo masculino, praticantes de futsal, caracterizando os grupos de prática sistematizada. Para a avaliação motora foram aplicados os testes de motricidade global e equilíbrio da Escala de Desenvolvimento Motor "EDM". Além disso, o questionário de atividade física habitual foi utilizado para calcular o gasto energético. A distribuição dos dados foi verificada através do teste Shapiro-Wilk, e em seguida foram aplicados os testes não-paramétricos Kruskall-Wallis com post hoc $U$ de Mann-Whitney, Wilcoxon e QuiQuadrado de Pearson. 0 nivel de significância foi estabelecido em $5 \%(p \leq 0,05)$. Resultados significantes foram encontrados entre os grupos de prática sistematizada com índices classificados como superiores e percentuais maiores em Idade Motora em Motricidade Global (IMMG) e Idade Motora em Equilíbrio (IME) do que idade cronológica (IC). Conclui-se que as crianças praticantes de atividades esportivas demonstraram superioridade nos testes aplicados quando comparados ao grupo controle, em que mais de 65\% apresentam classificação normal.

Palavras-chave: Atividade esportiva; Motricidade global; Equilibrio.

\section{Introdução}

A prática esportiva durante a infância vem se tornando alvo de pesquisas na área das ciências da motricidade humana, contribuindo de forma significativa para os estudos de crescimento físico, desenvolvimento e aprendizagem motora. Investigaçôes em atividades esportivas sistematizadas têm foco nos fatores ambientais, referentes à natureza da tarefa, a individualidade biológica e no que tange às explicaçôes das possíveis mudanças no desenvolvimento e nível de comportamento motor das crianças ${ }^{1-2}$.

Neste contexto, as práticas esportivas sistematizadas, de forma geral, contribuem significativamente para ampliaçáo do vocabulário motor das crianças. Assim, o presente estudo priorizou os aspectos de aprendizagem motora, trabalhando de forma diversificada todos os componentes da motricidade sem foco específico no aumento dos níveis de desempenho competitivo ${ }^{3}$. Entretanto, crianças, quando não são estimuladas adequadamente, podem não alcançar padróes de movimentos fundamentais para possível inserção em jogos, esportes e atividades recreativas ${ }^{4}$. Logo, quando a oferta de práticas e habilidades motoras fundamentais é reduzida pode, por consequência, ocorrer um prejuízo no curso do desenvolvimento motor da criança, ocasionando uma barreira intransponível, reconhecida na literatura como barreira de proficiência ${ }^{5}$. Crianças que náo apresentam um refinamento nas habilidades motoras fundamentais 
também não alcançam execução eficiente de movimento; sendo assim, até podem revelar interesse em atividades esportivas, contudo, apresentam grandes dificuldades com habilidades motoras requeridas e as adaptáveis às exigências do contexto ambiental ${ }^{4,6}$.

As aulas de Educação Física escolar, por sua vez, são precursoras em desenvolver todas as habilidades motoras fundamentais durante a infância, fornecendo práticas com objetivos definidos, respeitando as características do comportamento motor dos escolares ${ }^{7}$. Entretanto, estudos prévios têm demonstrado que em espaços extra-escolares é possível uma maior ampliação e aprimoramento do repertório motor ${ }^{8-10}$. Neste contexto e especificamente com relação ao "ballet" clássico, estudos apontam o efeito direto na orientação temporal, espacial, ritmicidade, flexibilidade e equilíbrio corporal dos praticantes ${ }^{11}$. Os autores ressaltam que durante a execução dos movimentos específicos do "ballet" clássico, as tarefas motoras exigem padróes de movimentos com amplitudes biomecanicamente elevadas. Desta forma, é notório

\section{Método}

\section{Participantes}

A amostra foi composta por 160 crianças, pré-púberes, eutróficas, na faixa etária de sete a 10 anos, subdivididas em quatro grupos equitativamente por sexo. Os grupos de escolares foram compostos por 80 participantes proveniente de escola pública, divididos em dois grupos, denominados escolares meninos e escolares meninas. Os grupos de prática sistematizada envolveram 80 crianças vinculadas a prática prévia de "ballet" clássico e futsal, com 40 meninas e meninos respectivamente, formando os dois grupos, futsal e "ballet". Após a localização dos grupos nas instituiçōes, foram realizadas reuniōes para explicaçôes dos procedimentos do estudo. Posteriormente a assinatura dos pais e/ou responsáveis legais do termo de consentimento livre e esclarecido, as avaliaçóes tiveram início. O Projeto de Pesquisa foi aprovado pelo Comitê de Ética e Pesquisa local (CEP/CGE n. 29/2010).

Os critérios de inclusão exigiram que, nos grupos de prática sistematizada, as crianças apresentassem experiência prévia mínima de um ano na modalidade esportiva, assiduidade mínima de duas vezes por semana e seis meses contínuas de prática antes do início das avaliaçóes. O grupo de futsal pertencia à Escolinha de Futsal Pequeno Craque, onde o objetivo da prática que a prática sistematizada influenciará na melhor projeção e execução dos gestos motores ${ }^{11-12}$. No caso do futsal, caracterizado por um amplo número de experiências locomotoras e manipulativas, a prática programada conduz a uma sincronia das funçóes musculares que agem diretamente na repercussão do melhor aparato motor ${ }^{13-14}$. Neste sentido, a prática de futsal e de "ballet" clássico deixam evidentes alguns parâmetros de habilidades locomotoras, manipulativas, capacidade de flexibilidade, ritmicidade e equilíbrio que são condizentes aos aspectos aqui avaliados $^{12-13}$. Portanto, hipoteticamente, crianças vinculadas exclusivamente à Educação Física escolar demonstraráo resultados de desenvolvimento motor abaixo do esperado quando comparados com praticantes de atividades programadas. Nesta direção, o presente estudo objetivou verificar o impacto da prática de atividades esportivas programadas prévias de "ballet" clássico e futsal sobre os parâmetros da motricidade global e equilíbrio de crianças de ambos os sexos entre sete a 10 anos. esportiva era trabalhar com foco na aprendizagem estruturada dos fundamentos específicos da modalidade, compreensão das regras e técnicas básicas do esporte. Para tanto, foram projetadas atividades recreativas e lúdicas para o melhor aprimoramento das habilidades motoras, respeitando as características de cada faixa etária. O grupo de "ballet" foi composto por meninas, pertencentes à Escola Municipal de Ballet, e as sessóes de prática eram realizadas duas vezes por semana no Polo de Ginástica Municipal, com objetivo de potencializar as estruturas rítmicas e coordenativas de diferentes partes do corpo e, principalmente, o aperfeiçoamento das capacidades físicas (força, velocidade, resistência e flexibilidade) e das habilidades motoras (saltitar, saltar, galopar, correr). O grupo de escolares foi composto por crianças matriculadas na Escola $\mathrm{Mu}$ nicipal Vera Cecília Lamin localizada no município de Jacarezinho - PR, vinculadas exclusivamente a aulas de Educação Física escolar, sendo realizadas duas vezes na semana. A escola tem quadra esportiva e materiais adequados para as aulas de Educação Física.

\section{Instrumentos e materiais}

Para obtenção de informaçóes relacionadas ao crescimento físico e ao estado nutricional das 
crianças foram realizadas medidas da massa corporal por uma balança eletrônica da marca Filizola com precisão de $0,1 \mathrm{~kg}$, e estatura mediante um estadiômetro de madeira com precisão de $0,1 \mathrm{~cm}$, estando à criança em posição ortostática de costas para a escala de medida, com os pés unidos no centro sobre a base do estadiômetro, com olhar dirigido para frente e sem calçados. De posse dos dados, foi calculado o índice de massa corporal (IMC), indicador nutricional que seguiu as recomendaçóes de classificação de eutrofia pelos percentis propostos pelo $\mathrm{CDC}^{15}$.

Posteriormente, foi investigado o nível de atividade física habitual mediante aplicação do recordatório do gasto energético diário proposto por BoUCHARD et al. ${ }^{16}$. Para obtenção destes resultados, um dos pesquisadores conduziu uma entrevista individual para explicar o questionário e auxiliar no preenchimento.

\section{Teste motor}

Para averiguar a caracterização do desenvolvimento motor foi utilizada a Escala de Desenvolvimento Motor $(\mathrm{EDM})^{2}$, evidenciada por um conjunto de testes em seis componentes da motricidade: motricidade global, motricidade fina, equilíbrio, esquema corporal, organização espacial, organização temporal e lateralidade. No presente estudo foram testadas a motricidade global e $\mathrm{o}$ equilíbrio, no sentido de atender as especificidades das modalidades investigadas. Cada componente da motricidade é composto por 10 tarefas motoras divididas por faixa etária, entre dois e 11 anos de idade, organizadas progressivamente em relação a sua complexidade, sendo que, quando a criança obtém êxito na tarefa é atribuído $\mathrm{o}$ valor da idade motora correspondente em meses. $\mathrm{O}$ teste é interrompido quando a criança não conclui a tarefa com sucesso, conforme os critérios estabelecidos pelo protocolo de avaliaçáo.

Essa bateria de avaliação motora determina a idade motora, obtida através dos pontos alcançados nos testes, e o quociente motor, resultado da divisão da idade cronológica multiplicado por 100 . Os valores referenciais de cada idade motora analisada são quantificados e categorizados, permitindo classificar o resultado em categorias que, nesse estudo, foram agrupadas: 1) muito inferior e inferior (menos de 69 até 79); 2) normal baixo e normal médio (de 80 a 109); e 3) normal alto, superior e muito superior (de 110 até acima de 130).

Os testes foram aplicados por um único avaliador treinado e experiente. A avaliação teve duração máxima de 20 minutos e foi conduzida de forma individual em ambiente fechado e exclusivo para avaliação no sentido de minimizar qualquer interferência externa.

\section{Análise dos dados}

Os dados foram submetidos ao teste de normalidade Shapiro Wilk e, por não seguirem distribuição normal, foram adotados testes estatísticos não paramétricos, com resultados expressos pelos valores da mediana e intervalo interquartílico. Foram consideradas como variáveis independentes os grupos ("ballet" clássico, futsal e escolares). As variáveis dependentes foram o gasto energético, as características antropométricas, idade motora nos testes de equilíbrio e de motricidade global. Para verificar as diferenças nas variáveis dependentes entre os grupos utilizou-se o teste Kruscall-Wallis, seguido de Mann-Whitney para a localização das diferenças. O teste de Wilcoxon foi empregado para verificar a magnitude da diferença entre cada par de observaçóes, neste caso, a comparaçáo entre as idades motoras e a idade cronológica. O teste Qui-quadrado de Pearson foi aplicado para testar a hipótese de que entre os grupos de prática esportiva (futsal e "ballet") se concentravam os maiores percentuais de crianças com idades motoras em equilíbrio e motricidade global classificadas como superiores. Posteriormente; utilizou-se o referido teste para analisar a distribuição das categorias da idade motora estratificadas em: 1) muito inferior e inferior; 2) normal baixo e normal médio; e 3) normal alto, superior e muito superior entre os grupos de escolares e os vinculados à prática esportiva sistematizada. Estas aplicaçôes do teste Qui-quadrado de Pearson permitiram compreender em quais grupos estavam as maiores distribuiçóes das idades motoras superiores. Para todas as análises foi adotada significância estatística de $\mathrm{p} \leq 0,05$.

\section{Resultados}

A TABELA 1 demonstra as características antropométricas e gasto energético dos grupos estudados.
Os resultados demonstraram homogeneidade entre os grupos para as variáveis de idade cronológica, massa corporal total, estatura e IMC. Seguindo os 
Teste de Kruskal-Wallis seguido de Teste "U" de Mann-Whitney para localização das diferenças.

IC = idade cronológica; $\mathrm{MC}=$ massa corporal; IMC = índice de massa corporal;

$\mathrm{GE}=$ gasto energético. a GE maior entre 0 grupo de futsal em relação ao grupo "ballet" e escolares meninas ( $p$ $=0,005$ )

b GE maior entre o grupo futsal em relação ao grupo escolares meni$\operatorname{nos}(p=0,022)$

Teste de Associação (Qui-quadrado de Pearson- $X^{2}$ );

Grupo Masculino (escolares e futsal) $-p=$ 0,001

Grupo Feminino (escolares e "ballet") $-p=$ 0,0001 .

critérios de inclusão, todas as crianças apresentaram eutrofia de acordo com os critérios do $\mathrm{CDC}^{15}$. As diferenças significativas foram localizadas somente no gasto energético, demonstrando valores superiores entre os meninos do futsal (mediana $1351 \mathrm{kcal}$; $\mathrm{p}=0,005)$ quando comparados com seu respectivo grupo de escolares (mediana $1197 \mathrm{kcal} ; \mathrm{p}=0,022$ ). Os valores do grupo futsal também foram superiores ao do grupo escolares do sexo feminino (mediana 1089 kcal; $\mathrm{p}=0$,005) e do grupo de "ballet" clássico (mediana $1175 \mathrm{kcal} ; \mathrm{p}=0,005$ ).

TABELA 1 - Mediana (M) e intervalo interquartílico (Q1-Q3) das variáveis de crescimento físico e estimativa de GE dos grupos investigados.

\begin{tabular}{|c|c|c|c|c|}
\hline & $\begin{array}{l}\text { Escolares meninos } \\
\qquad M(Q 1 ; Q 3)\end{array}$ & $\begin{array}{c}\text { Futsal } \\
M(Q 1 ; Q 3)\end{array}$ & $\begin{array}{l}\text { Escolares meninas } \\
\qquad \mathrm{M}(\mathrm{Q} 1 ; \mathbf{Q} 3)\end{array}$ & $\begin{array}{c}\text { "Ballet" } \\
\text { M(Q1;Q3) }\end{array}$ \\
\hline IC (anos) & $8,5(7,3-9,1)$ & $8,5(7,7-9,7)$ & $8,5(8,0-9,1)$ & $8,5(7,7-9,7)$ \\
\hline $\mathrm{MC}(\mathrm{kg})$ & $31,0(27,0-31,6)$ & $34,5(27,1-43,2)$ & $30,3(26,2-40,4)$ & $31,9(26,8-38,1)$ \\
\hline Estatura $(\mathrm{m})$ & $1,35(1,30-141)$ & $1,36(1,32-1,44)$ & $1,32(1,27-142)$ & $1,37(1,29-1,44)$ \\
\hline $\operatorname{IMC}\left(\mathrm{kg} / \mathrm{m}^{2}\right)$ & $17,2(15,4-19,8)$ & $18,4(15,6-21,0)$ & $17,5(15,6-20,5)$ & $16,4(15,4-18,6)$ \\
\hline GE (kcal/dia) & $1197(995-1410)^{\mathrm{b}}$ & $1351(1093-1630)^{\mathrm{a}, \mathrm{b}}$ & $1089(934-1449)^{\mathrm{a}}$ & $1175(972-1414)^{a}$ \\
\hline
\end{tabular}

TABELA 2 - Associação entre as classificações Inferior, Normal e Superior da Escala de Desenvolvimento Motor para Idade Motora em Motricidade Global (IMMG) nos grupos de treinamento e escolares.

\begin{tabular}{lcccccccc}
\hline & $\begin{array}{c}\text { Escolares meninos } \\
(\mathbf{n = 4 0 )}\end{array}$ & \multicolumn{2}{c}{$\begin{array}{c}\text { Futsal } \\
(\mathbf{n = 4 0 )}\end{array}$} & $\begin{array}{c}\text { Escolares meninas } \\
(\mathbf{n = 4 0 )}\end{array}$ & \multicolumn{2}{c}{$\begin{array}{c}\text { "Ballet" } \\
(\mathbf{n}=\mathbf{4 0})\end{array}$} \\
\hline & $\mathrm{f}$ & $\%$ & $\mathrm{f}$ & $\%$ & $\mathrm{f}$ & $\%$ & $\mathrm{f}$ & $\%$ \\
Inferior & 1 & 2,5 & - & - & 2 & 5 & - & - \\
Normal & 31 & 77,5 & 18 & 45 & 30 & 75 & 11 & 27 \\
Superior & 8 & 20,0 & 22 & 55 & 8 & 20 & 29 & 78 \\
\hline
\end{tabular}

A TABELA 3 demonstra associação significante entre a classificação de Idade Motora em Equilíbrio (IME) e os grupos avaliados, onde $65 \%$ do grupo de "ballet" clássico demonstraram valores classificados como superior, enquanto as escolares apresentaram percentuais elevados de $82,5 \%$ na classificação normal $\left(\mathrm{X}^{2}=18,387 ; \mathrm{p}=0,01\right)$, indicando a potencialização do esporte para essa habilidade. $\mathrm{O}$ comportamento foi similar no sexo masculino, com $65 \%$ dos meninos do grupo escolar classificados na categoria normal e $50 \%$ dos praticantes de futsal indicaram valores significantes classificados como normal ou superior $\left(\mathrm{X}^{2}=5,876 ; \mathrm{p}<0,05\right)$.
A TABELA 2 destaca os resultados do teste de associação entre a classificação de Idade Motora em Motricidade Global (IMMG) e os grupos avaliados. Os resultados apontaram que aproximadamente $76 \%$ dos escolares obtiveram desempenho classificado como normal. Nos grupos de prática sistematizada, $50 \%$ das crianças vinculadas ao "ballet" clássico e ao futsal apresentaram valores na classificação superior $\left(X^{2}=10,845 ; p=0,001\right.$ entre escolares do sexo masculino e futsal e $X^{2}=22,053 ; p=0,0001$ entre escolares do sexo feminino e "ballet" clássico). 
TABELA 3 - Associação entre as classificações Inferior, normal e superior da Escala de Desenvolvimento Motor para Idade Motora em Equilíbrio (IME) nos grupos.

\begin{tabular}{|c|c|c|c|c|c|c|c|c|}
\hline & \multicolumn{2}{|c|}{$\begin{array}{l}\text { Escolares meninos } \\
\qquad(\mathrm{n}=40)\end{array}$} & \multicolumn{2}{|c|}{$\begin{array}{c}\text { Futsal } \\
(n=40)\end{array}$} & \multicolumn{2}{|c|}{$\begin{array}{l}\text { Escolares meninas } \\
\qquad(\mathrm{n}=40)\end{array}$} & \multicolumn{2}{|c|}{$\begin{array}{l}\text { "Ballet" } \\
(\mathrm{n}=40)\end{array}$} \\
\hline & $\mathrm{f}$ & $\%$ & $\mathrm{f}$ & $\%$ & $\mathrm{f}$ & $\%$ & $\mathrm{f}$ & $\%$ \\
\hline Inferior & 7 & 7,5 & - & - & - & - & - & - \\
\hline Normal & 26 & 65,0 & 20 & 50 & 33 & 82,5 & 14 & 35 \\
\hline Superior & 11 & 27,5 & 20 & 50 & 7 & 17,5 & 26 & 65 \\
\hline
\end{tabular}

TABELA 4 - Frequência absoluta erelativa para os quatro grupos das relações entrea Idade Motora em equilíbrio (IME) e a Idade Cronológica (IC).

\begin{tabular}{lcccc}
\hline & IME<IC & IME>IC & IME=IC & \\
& $\mathbf{f ( \% )}$ & $\mathbf{f}(\mathbf{\%})$ & $\mathbf{f}(\mathbf{\%})$ & $\mathbf{p}$ \\
\hline Escolares meninos & $24(60,0)$ & $12(30,0)$ & $4(10,0)$ & 0,120 \\
Futsal & $9(22,5)$ & $29(72,5)$ & $2(5,0)$ & $0,0001^{*}$ \\
Escolares meninas & $25(62,5)$ & $14(35,0)$ & $1(2,5)$ & 0,346 \\
"Ballet” & $9(22,5)$ & $31(77,5)$ & - & $0,0001^{*}$ \\
\hline
\end{tabular}

TABELA 5 - Frequência absoluta e relativa para os quatro grupos das relações entre Idade Motora em Motricidade Global (IMMG) e Idade Cronológica (IC).

\begin{tabular}{lcccc}
\hline & IMMG $<$ IC & IMMG $>$ IC & IMMG=IC & \\
& $\mathbf{f ~ ( \% )}$ & $\mathbf{f}(\mathbf{\%})$ & $\mathbf{f ~ ( \% )}$ & $\mathbf{p}$ \\
\hline Escolares meninos & $14(35,0)$ & $21(52,5)$ & $5(12,5)$ & 0,528 \\
Futsal & $4(10,0)$ & $33(82,5)$ & $3(7,0)$ & $0,0001^{*}$ \\
Escolares meninas & $21(52,5)$ & $17(42,5)$ & $2(5,0)$ & 0,384 \\
"Ballet" & $4(10,0)$ & $36(90,0)$ & - & $0,0001^{*}$ \\
\hline
\end{tabular}

\section{Discussão}

O presente estudo, ao avaliar crianças de sete a 10 anos de idade em cenários distintos, verificou que os praticantes de ambos os sexos vinculados às atividades esportivas demonstraram relaçóes de motricidade global e equilíbrio em escores superiores (> 50\%) quando comparados com as crianças pertencentes exclusivamente a Educação Física escolar.

A faixa etária avaliada no presente estudo foi caracterizada como segunda infância e, neste sentido, destacam-se o potencial de desenvolvimento motor da maioria dos movimentos fundamentais, iniciando um processo de transição para uma etapa especializada posterior. Assim, um ambiente que favoreça o aprimoramento motor e proporcione estímulos para o desenvolvimento dos componentes da motricidade tem potencial de auxiliar no domínio do controle motor e maximizar o desenvolvimento motor ${ }^{4}$.

Em relação à comparação do gasto energético, verificou-se que os meninos praticantes de futsal apresentaram valores superiores aos demais. Neste contexto, é reconhecido que o exercício físico promove o aumento da demanda energética quando comparado com a situaçáo de repouso, evidenciando que o gasto de energia sofre variação com a frequência, a duração e com a intensidade das atividades exigidas pelos esportes ${ }^{17}$. Este resultado indica que as crianças participantes de atividades com volume e intensidade maiores de treinamento, como é o caso do futsal no presente estudo, demonstram um gasto energético maior que
Teste de Wilcoxon; ${ }^{*} p<0,05$.
Teste de Wilcoxon; ${ }^{*} p<0,05$. de Associação Qui-quadrado de Pearson - $X^{2}$ ) Grupo Masculino (escoares e futsal) $-p<0,05$ Grupo Feminino (escolares e "ballet") - $p$ $=0,01$.



(1) 
as crianças que praticam atividades mais moderadas, como comparativamente no "ballet" clássico.

Com relação à programação de protocolos de práticas sistematizadas na infância, recentemente MYER et al. ${ }^{18}$, ressaltaram a importância dos componentes maturacionais como indicadores preditivos para a base de um trabalho de atividades programadas. No presente estudo, todos os participantes incluídos estavam em estágio pré-pubertário, sem qualquer indicativo de características sexuais secundárias. A seleção exclusiva de pré-púberes neste estudo minimizou vieses nos resultados, uma vez que não houve qualquer contaminação no desempenho dos testes provenientes do avanço da maturação biológica. É reconhecido que o acompanhamento de indicadores biológicos e instruçóes adequadas nos programas sistematizados fazem com que a prática de atividades esportivas com crianças e adolescentes seja efetuada de maneira satisfatória, minimizando o riscos e lesóes aos praticantes ${ }^{19-20}$.

Os resultados desta pesquisa demonstraram que as crianças vinculadas à prática esportiva obtiveram superioridade na IMMG e IME quando comparados com os grupos compostos por escolares, indicados nas TABELAS 2 e 3. Neste sentido, os resultados demonstraram que a hipótese do teste de associação foi verdadeira, tendo evidenciado valores percentuais superiores em IMMG e IME nos grupos de prática esportiva sistematizada. Corroborando com nossos achados, Braga et al. ${ }^{10}$ e Cotrim et al. ${ }^{9}$, ambos investigando o contexto escolar realizaram a aplicação do Test of Gross Motor Development (TGMD-2), sendo que no primeiro estudo o objetivo foi verificar as diferenças entre grupos de escolares com atividades de intervenção específica ao TGMD-2 e o segundo foi analisar as diferenças no perfil motor em crianças inseridas em condiçôes formais de aulas de Educação Física (ministrada ou náo pelo professor da área, com e sem espaço físico e materiais). Braga et al. ${ }^{10}$ verificaram que as crianças participantes da intervenção, especificamente nas seis sub-áreas locomotoras da bateria TGMD-2 (corrida, galope, salto com um pé, passada, salto horizontal e corrida lateral) apresentaram níveis superiores quando comparados com o grupo não ativo. $\mathrm{O}$ mesmo resultado foi verificado no estudo conduzido por СотRIм et al. ${ }^{9}$, no qual as crianças que tiveram o direcionamento formal de um professor de Educação Física se sobressaíram sobre o grupo que não tinha condiçôes formais de Educação Física. Esses dados demonstram que apenas os fatores biológicos não garantem um desenvolvimento motor pleno, mesmo se tratando das habilidades motoras fundamentais, e que o direcionamento estruturado, sendo este por intervenção ou por acompanhamento formal do professor de Educação Física provoca alteraçóes no curso do desenvolvimento motor.

No presente estudo, os valores de referência na classificação da escala de desenvolvimento motor assinalam que, em todas as idades dos escolares, observou-se um padrão normal, com percentual superior a 65\% nas IMMG e IME. Este resultado indica que o ambiente escolar estimulou as capacidades coordenativas, mas sugere que neste processo algumas crianças podem ficar estagnadas, não alcançando aprimoramento do padrão maduro de movimento ${ }^{4}$. Em contrapartida aos níveis de coordenação normal dos escolares, os grupos de prática sistematizada apresentaram percentuais altos na classificação superior em todas as idades motoras, sendo na IME e IMMG e na frequência absoluta e relativa daqueles que apresentaram IME e IMMG superior à idade cronológica (TABELAS 4 e 5).

De forma similar aos resultados do estudo, em relação aos níveis de coordenação dos escolares e enfatizando a importância de atividades extra-escolares, Araújo et al. ${ }^{8}$, investigaram a participação de crianças em atividades extracurriculares com esportes radicais. O protocolo experimental foi dividido em três aulas semanais com atividades de "skate", patins, escalada e "parkour". Com aplicaçáo da bateria de testes TGMD-2 foram identificadas que as crianças que participaram das atividades extracurriculares apresentaram valores de desempenho maiores nas habilidades locomotoras e de controle de objetos do que as do grupo que não tinha práticas extra-escolares. Entretanto, tal como o grupo escolar do presente estudo, os resultados do grupo exclusivo de Educação Física apresentaram classificação dentro dos padróes normais. Os autores declararam que as diferenças desenvolvimentistas estavam interligadas com as experiências prévias dos indivíduos e é responsabilidade do professor de Educação Física escolar oferecer um ambiente de prática organizado e estruturado, otimizando o repertório motor dos seus alunos ${ }^{8}$. Nesta direção, com relação ao sexo feminino, o presente estudo constatou que mais de $50 \%$ das meninas do "ballet" clássico apresentaram índices superiores na classificação em todas as idades avaliadas, náo ocorrendo o mesmo com o grupo de escolares que demonstrou índices normais tanto na IME como IMMG. Este resultado pode ser explicado pela especificidade do "ballet" clássico e em outras modalidades de dança, nas quais a sustentação e a postura são componentes fundamentais para o equilíbrio e o desempenho geral da modalidade ${ }^{21}$. O equilíbrio é uma capacidade 
crucial para o desempenho no "ballet" clássico, tendo sido reportado em estudo envolvendo bailarinas clássicas, modernas e jogadoras de futebol altamente treinadas $^{22}$. Além disso, é reconhecido que esportes como futebol também demandam de equilíbrio para a maioria das jogadas, nas mudanças de direção em altas velocidades e nas finalizaçóes, sendo assim, foram comparadas condiçôes de controle postural, entre os grupos de bailarinas clássicas, modernas e jogadoras de futebol. As medidas utilizadas foram de equilíbrio unipodal (composto pela análise feita com olhos abertos, fechados e análises de saltos) utilizando como instrumento uma plataforma de força. Foram verificadas, na maioria das análises, que o grupo das bailarinas, tanto na modalidade clássica como moderna demonstraram menores oscilaçóes no centro de pressão quando comparados com jogadoras de futebol. Os autores ressaltam que os resultados estão estritamente relacionados com a especificidade da prática do treinamento de "ballet", deixando claro que dentro das variáveis de equilíbrio analisadas as bailarinas tiveram maior êxito ${ }^{22}$.

Estes resultados reforçam os posicionamentos de Bruyneel et al. ${ }^{23}$ que examinaram diferenças no equilíbrio entre grupos de jovens entre oito a 16 anos e adultos entre 17 e 30 anos, todos praticantes de "ballet", utilizando plataforma de força. Os resultados sinalizaram que adultos com maior tempo de prática apresentaram menores oscilaçóes nos balanços (ântero-posterior) ( $\mathrm{p}<0,05)$. Os autores afirmam que, além do período de prática, um período médio de dança influencia na capacidade de antecipação e no realinhamento do equilíbrio de acordo com as sugestóes perceptivas, corroborando com os resultados do presente estudo.

Verificando a melhora das habilidades motoras com a prática de "ballet", SouzA et al. ${ }^{24}$, avaliaram grupos de meninas de sete a 10 anos, por meio do TGMD-2, com intervenção de: a) habilidades locomotoras fundamentais (corridas, saltos e galope); b) habilidades manipulativas fundamentais (rebatida, quique, recepção, arremesso e rolo de bola); c) habilidades motoras especializadas da dança ("Grand Jeté" - salto do "ballet" e "jazz" e, "Chassé" - deslocamento do "ballet" clássico). Os resultados revelaram que após a intervenção, as meninas apresentaram ganhos significativos $(p \leq 0,05)$ nas habilidades de passada e galope, evidenciando que a prática sistematizada de dança potencializou o desenvolvimento de habilidades motoras fundamentais e especializadas.

Os resultados em relação à prática de futsal apresentaram resultados similares ao do "ballet" clássico onde aproximadamente $50 \%$ dos meninos do grupo futsal do presente estudo apresentaram níveis superiores quando comparados com o grupo escolar (TABELAS 2 e 3). Além disso, é importante destacar que o grupo escolar demonstrou um valor percentual acima na classificação normal tanto na IMMG como na IME. De forma similar, NAZARio e Vieira ${ }^{25}$ analisaram atividades programadas de ginástica, futsal e handebol em crianças nas faixas etárias de sete a 10 anos e compararam com crianças que participavam exclusivamente das aulas de Educação Física escolar. Aplicação do teste TGMD-2 apontou as diferenças nas habilidades correspondentes com a prática de cada modalidade, no caso do futsal os testes de chute e corrida lateral apontaram resultados superiores $(\mathrm{p}<0,001)$ quando comparados entre as modalidades, assim como os testes de saltar com um pé e arremesso por cima do ombro as crianças do handebol demonstraram valores superiores aos demais. Dentro dos escores da classificação do TGMD-2, as crianças participantes apenas de Educação Física ficaram classificadas como "muito pobre", no entanto, crianças vinculadas às modalidades de ginástica e handebol se classificaram como "abaixo da média” e o futsal na "média". De maneira geral, os autores concluíram que as crianças inseridas em ambientes esportivos apresentam perfil motor específico resultante das exigências da modalidade.

Investigando especificamente a modalidade de futsal, Rocha et al. ${ }^{26}$, verificaram o efeito do treinamento sobre o desenvolvimento motor de crianças de seis a nove anos. Foram divididos dois grupos da mesma faixa etária, um grupo iniciante e outro de praticantes de futsal há seis meses, todos foram avaliados pela escala de desenvolvimento motor proposta por Rosa $\mathrm{NeTO}^{2}$. Os resultados indicaram que os iniciantes não apresentaram índices abaixo da classificação normal, enquanto os praticantes com tempo superior a seis meses demonstraram valores significantes $(\mathrm{p}<0,05)$ na classificação normal alto. Esses achados reforçam que a prática esportiva, mesmo que por pouco tempo (seis meses), contribuiu positivamente para a potencialização do desenvolvimento motor nas habilidades de motricidade fina, motricidade global e esquema corporal.

Outras investigaçóes avaliaram diversos elementos da prática habitual de atividade física, testes de aptidão física, aplicação de questionários e baterias de avaliação motora (Körperkoordination-TestfürKinder-KTK; Champs Motor Skill Protocol-CMSP). Os resultados indicaram que crianças que tem um 
desempenho baixo de habilidades locomotoras, não alcançando padrōes fundamentais do movimento, são fisicamente menos ativas do que crianças com habilidades motoras mais desenvolvidas. Neste sentido, um trabalho inicial é primordial para elevar os índices de inserção das crianças em programas de atividades sistematizadas, promovendo um método de prevenção à saúde além de um estímulo futuro para se tornarem ativos quando adultos ${ }^{27-29}$.

Por fim, de maneira geral, há o entendimento de que atividades programadas, sejam elas específicas ou gerais, podem ser determinantes quando se busca uma pré-estruturação dos níveis de comportamento motor, revelando que se bem trabalhadas maximizam a velocidade e a facilidade do aprendizado de novas habilidades, assim como a progressão para as habilidades especializadas $s^{30-32}$.
Conclui-se que crianças praticantes de atividades esportivas extra-curriculares apresentam níveis superiores de motricidade global e equilíbrio, com coeficiente motor de classificação normal a superior. Por outro lado, no ambiente escolar, os resultados da idade motora global e equilíbrio apresentaram índices normais. Assim, crianças vinculadas exclusivamente às aulas de Educação Física escolar do presente estudo náo demonstram déficits no desempenho dos testes aplicados apontando que a hipótese de níveis baixos de desenvolvimento motor foi refutada.

Um dos pontos limitantes do presente estudo se refere à ausência de uma análise mais detalhada das aulas de Educação Física e dos treinamentos sistematizados. Assim, em estudos futuros se recomenda o acompanhamento e a descrição minuciosa do treinamento e das atividades realizadas nas aulas de Educação Física.

\begin{abstract}
The effect of systematic sports activities on the motor development of children from seven to 10 years

It is recognized that systematic physical activities cause changes in many aspects of children's development. Thus, the present study aimed to verify the impact of programmed sports activities, classical ballet and futsal, on indicators of global motor function and balance in children. The sample consisted of 160 children between 7 and 10 years of age. Eighty school children of both sexes were selected, characterized by the exclusive practice of school Physical Education. The programmed sports groups were composed of 40 female children, classical ballet practitioners, and 40 males who participated in futsal, characterizing the systematized practice group. The Motor Development Scale MDS was applied to assess global motor function and balance. In addition, the habitual physical activity questionnaire was used to calculate energy expenditure. Data distribution was verified using the Shapiro-Wilk's test and then were applied non-parametric tests of the Kruskall-Wallis test with post hoc Mann-Whitney U, Wilcoxon and Chi-Square Pearson tests. The significance level was set at 5\% ( $p \leq 0.05)$. Significant results were found in the systematized practice groups, with indices classified as superior and higher percentage for Motor Age of Global Motricity (MAGM) and Motor Age of Balance (MAB), when compared to chronological age (CA). In conclusion the children who practiced programmed sports activities demonstrated superiority in the tests when compared to the control group, where more than 65\% were classified as normal.
\end{abstract}

KEY WORDS: Sports activity; Global motor; Balance.

\title{
Referências
}

1. Wrotniak BH, Epstein LH, Dorn JM, Jones KE, Kondilis VA. The relationship between motor proficiency and physical activity in children. Pediatrics. 2006;118:1758-65.

2. Rosa Neto F. Manual de avaliação motora. Porto Alegre: Artmed; 2002.

3. Fransen J, Pion J, Vandendriessche J, et al. Differences in physical fitness and gross motor coordination in boys aged 6-12 years specializing in one versus sampling more than one sport. J Sports Science. 2012;30:379-86.

4. Gallahue D, Donnelly FC. Educação física desenvolvimentista para todas as crianças. São Paulo: Phorte; 2008. 
5. Seefeldt V, Malina RM, Clark MA. Factors affecting levels of psysical activity in adults. Sports Med. 2002;32:143-68.

6. Clark JE. Motor development. Enc Hum Behavior. 1994,3:245-55.

7. Tani G. Abordagem desenvolvimentista: 20 anos depois. Rev Ed Fís/UEM. 2008;19:313-31.

8. Araújo MP, Barela JA, Celestino ML, Barela AMF. Contribuição de diferentes conteúdos das aulas de educação física no ensino fundamental I para o desenvolvimento das habilidades motoras fundamentais. Rev Bras Med Esporte. 2012;18:153-7.

9. Cotrim JR, Lemos AG, Neri Júnior JE, Barela JA. Desenvolvimento das habilidades motoras fundamentais em crianças com diferentes contextos escolares. Rev Ed Fís/UEM. 2011;22:523-33.

10. Braga RK, Krebs RJ, Valentini NC, Tkac CM. A influência de um programa de intervenção motora no desempenho das habilidades locomotoras de crianças com idade entre 6 e 7 anos. Rev Ed Fís/UEM. 2009;20:171-81.

11. Prati SRA, Prati ARC. Níveis de aptidáo física e análise de tendências posturais em bailarinas clássicas. Rev Bras Cineantropom Desempenho Hum. 2006;8:80-7.

12. Meereis, ECW. Análise de tendências posturais em praticantes de balé clássico. Rev Ed Fís/UEM. 2011;1:27-35.

13. Buzolin Neto O, Barbieri FA, Barbieri RA, Gobbi LTB. Agility, speed and motor skill performance of practitioners and non-practitioners of soccer. Fit Perform J. 2009;8:110-4.

14. Mortatti AL, Arruda M. Análise do efeito do treinamento e da maturação sexual sobre o somatotipo de jovens futebolistas. Rev Bras Cineantropom Desempenho Hum. 2007;9:84-91.

15. Kuczmarski RJ, Ogden CL, Grummer SLM, et al. CDC growth charts: United States. Adv Data. 2000;314:1-27.

16. Bouchard, CA, Tremblay A, Leblanc C, Lortie G, Savard R, Thériault G. Method to assess energy expenditure in children and adults. Am J Clin Nutr. 1983;37:461-7.

17. American College of Sports Medicine. Diretrizes do ACSM para os testes de esforço e sua prescrição. Rio de Janeiro: Guanabara Koogan; 2003.

18. Myer GD, Lloyd, RS, Brent, JL, Faigenbaum, AD. How young is "too young” to start training? ACSM's Health Fit J. 2013;17:14-23.

19. Myer, GD, Kushner, AM, Faigenbaum AD, Kiefer A, Kashikar_Zuck S, Clark JF. Training the developing brain, part I: cognitive developmental considerations for training youth. Curr Sports Med Rep. 2013;12:304-10.

20. Lloyd RS, Oliver JL, Meyers RW, Moody JA, Stone, MH. Long-term athletic development and its application to youth weightlifting. Strength Cond J. 2012;34:55-66.

21. Costa PHL, Nora FGSA, Vieira MF, Bosch K. Single leg balancing in ballet: effects of shoe conditions and poses. Gait Posture. 2013;37:419-23.

22. Gerbino PG, Griffin ED, Zurakowski D. Comparison of standing balance between female collegiate dancers and soccer players. Gait Posture. 2007;26:501-7.

23. Bruyneel AV, Mesure S, Paré JC, Bertrand M. Organization of postural equilibrium in several planes in ballet dancers. Neurosci Lett. 2010;485:228-32.

24. Souza MC, Berleze A, Valentini NC. Efeitos de um programa de educação pelo esporte no domínio das habilidades motoras fundamentais e especializadas: ênfase na dança. Rev Ed Fís/UEM. 2008;19:509-19.

25. Nazario PF, Vieira JLL. Sport context and the motor development of children. Rev Bras Cineantropom Desempenho Hum. 2010;16:86-95.

26. Rocha PGM, Rocha DJO, Bertolasce AL. A influência da iniciação ao treinamento esportivo sobre o desenvolvimento motor na infância: um estudo de caso. Rev Ed Fís/UEM. 2010;21:469-77.

27. Carter CW, Micheli LJ. Training the child athlete: physical fitness, health and injury. Brit J Sports Med. 2011;45:880-85.

28. Lopes VP, Rodrigues LP, Maia JAR, Malina RM. Motor coordination as predictor of physical activity in childhood. Scand J Med Sci Sports. 2011;21:663-9.

29. Williams HG, Pfeiffer KA, O'neill JR, et al. Motor skill performance and physical activity in preschool children. Obesity. 2008;16:1421-6.

30. Gimenez R, Manoel EJ, Oliveira DL, Dantas L, Marques I. Integrating fundamental movement skills in late childhood. Percept Mot Skills. 2012;114:563-83.

31. Raudsepp L, Pall P. The relationship between fundamental motor skills and outside-school physical activity of elementary school. Pediatr Exerc Sci. 2006;18:426-35.

32. Okely AD, Booth ML, Patterson JW. Relationship of physical activity to fundamental movement skills among adolescents. Med Sci Sports Exerc. 2001;33:1899-904. 
